\title{
High-performance elastocaloric materials for the engineering of bulk- and micro-cooling devices
}

\author{
Jan Frenzel, Gunther Eggeler, Eckhard Quandt, Stefan Seelecke, \\ and Manfred Kohl
}

Pseudoelastic NiTi-based shape-memory alloys (SMAs) have recently received attention as candidate materials for solid-state refrigeration. The elastocaloric effect in SMAs exploits stress-induced martensitic transformation, which is associated with large latent heat. Most importantly, cyclic mechanical loading/unloading provides large adiabatic temperature drops exceeding $25 \mathrm{~K}$ at high process efficiencies. This article summarizes the underlying principles, important material parameters and process requirements, and reviews recent progress in the development of pseudoelastic SMAs with large coefficients of performance, as well as very good functional fatigue resistance. The application potential of SMA film and bulk materials is demonstrated for the case of cyclic tensile loading/unloading in prototypes ranging from miniature-scale devices to large-scale cooling units.

\section{Introduction}

Refrigeration and cooling account for a significant fraction of global energy usage. The need for reduction of energy consumption and greenhouse gas emissions has motivated research in new solid-state cooling concepts (ferroic cooling). ${ }^{1}$ These include magneto-, electro-, or elastocaloric effects. Pseudoelastic NiTi-based shape-memory alloys (SMAs) are a very promising class of candidate materials for elastocaloric cooling. ${ }^{2,3}$ While NiTi SMAs are better known for being able to restore their initial geometry after large deformations, ${ }^{4-6}$ these materials have recently also been identified as a promising solid-state alternative to well-established vapor compression cooling systems. ${ }^{7}$

The elastocaloric effect (i.e., pseudoelasticity, a mechanical shape-memory behavior) relies on a reversible martensitic phase transformation, ${ }^{4-6}$ a specific type of solid-state transformation. It represents a shear process in the crystal lattice, ${ }^{8}$ and can be triggered by cooling or mechanical loading. Figure 1 shows a schematic elastocaloric cooling cycle starting at the high-temperature austenite state, stage 0 in Figure 1a. During mechanical loading, a stress/strain plateau is reached where the parent phase, austenite, transforms into stress-induced martensite, stage 1 to stage 2 . The reverse transformation, stage 3 to stage 4 , is characterized by an unloading plateau at lower stresses. Both transformation events are associated with large latent heat $\Delta H$, and thus cause remarkable temperature changes in the SMA.

The area, $W$, in Figure 1a represents the mechanical work input (i.e., force and distance) required to drive an ideal cooling cycle. It is common to characterize the cooling efficiency by the coefficient of performance (COP), which represents the ratio between the cooling energy output $\Delta H$ and the required work input, $W .{ }^{9}$ Figure $1 \mathrm{~b}$ shows different material stages of the cooling cycle. The forward phase transformation that occurs during loading between stages 1 and 2 results in the release of latent heat and thus in an increase in the SMA temperature. This heat amount needs to be absorbed by a heat sink, such that a lower temperature is again approached in the material. During unloading, the reverse transformation causes a significant temperature drop between stages 3 and 4 . This drop, which corresponds to values between $15^{\circ} \mathrm{C}$ and $30^{\circ} \mathrm{C}$ for $\mathrm{NiTi},{ }^{9-11}$ determines the lowest achievable temperature in a ferroic cooling process.

Present research on elastocaloric cooling follows two main directions. First, basic aspects related to the material behavior, such as heat effects, functional stability, and alloy design, are

Jan Frenzel, Ruhr University Bochum, Germany; jan.a.frenzel@rub.de

Gunther Eggeler, Ruhr University Bochum, Germany; gunther.eggeler@rub.de

Eckhard Quandt, Kiel University, Germany; eq@tf.uni-kiel.de

Stefan Seelecke, Saarland University, Germany; stefan.seelecke@imsl.uni-saarland.de

Manfred Kohl, Institute of Microstructure Technology, Karlsruhe Institute of Technology, Germany; manfred.kohl@kit.edu

doi:10.1557/mrs.2018.67 

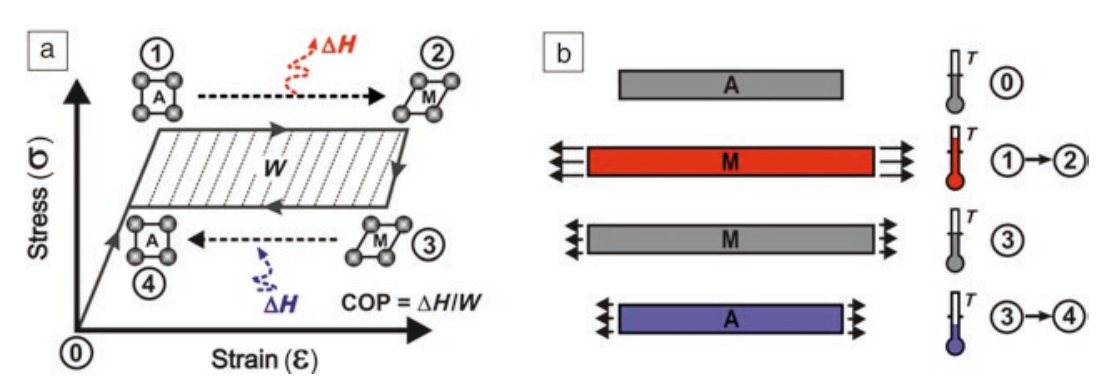

of small hysteresis width and large latent heat simultaneously. Nevertheless, Wieczorek et al. ${ }^{10}$ recently found a good compromise in a $\mathrm{NiTiCuV}$ SMA with an exceptionally high COP value of 20 . This value is about $4 \times$ higher than for binary NiTi. ${ }^{9}$ Schmidt et al..$^{30}$ made a first attempt to study the cooling performance of this material.

Figure 1. Heat effects in pseudoelastic shape-memory alloys (SMAs). (a) Stress/strain behavior of the material. The martensitic transformation during loading results in the release of latent heat and thus increases the SMA temperature. During the reverse transformation on unloading, a temperature drop causes absorption of latent heat $(\Delta H)$. $\mathrm{COP}$ is coefficient of performance; the area $W$ represents the mechanical work input required to drive an ideal cooling cycle. (b) Different material stages of an elastocaloric cooling cycle. Note: A, austenite; M, martensite.

being investigated. ${ }^{9-14}$ Second, the use of solid-state materials in a cooling process requires new engineering approaches which are being explored. ${ }^{915-18}$ Thus, different process design concepts are being developed and evaluated. In the present article, we give a brief overview of recent progress and challenges in these interesting research fields. We consider the case of tensile loading, which allows for high-aspect ratios of the material and thus fast heat exchange as compared to compressive loading. On the other hand, tensile loading also facilitates the formation and growth of fatigue cracks. Nevertheless, this risk can be tackled by using suitable alloy compositions ${ }^{19}$ and material states with low defects densities. ${ }^{20}$

\section{Elastocaloric material behavior Latent heat and hysteresis width}

Four material parameters ${ }^{9,10}$ are important for elastocaloric cooling performance. The SMA must exhibit large latent heat $\Delta H$; low hysteresis width, which corresponds to a low work input, $W$; and good functional and structural stability, sufficient for several years of service life $\left(>10^{7}\right.$ loading/ unloading cycles). It also must be able to deliver the elastocaloric effect in an envisaged temperature regime.

It is well known that the martensitic transformation and thus pseudoelastic behavior is governed by alloy composition $^{10,14}$ and microstructure. ${ }^{21,22}$ Various research groups $s^{10,11,15}$ have made attempts to study the effects of alloy chemistry on cooling performance. Both latent heat and hysteresis width react sensitively to compositional changes. ${ }^{10,23-29}$ Latent heats between 8.5 and $35 \mathrm{~J} / \mathrm{g},{ }^{23}$ and thermal hysteresis widths ranging from $\approx 0 \mathrm{~K}$ to $>70 \mathrm{~K},,^{23,26}$ are reported in the literature. We note that the thermal hysteresis width, which is often used as a preferred indicator in compositional screenings, ${ }^{25,26}$ is directly proportional to the mechanical hysteresis width ${ }^{27}$ and thus to the required input work, $W$ (Figure 1a). The results of a recent study ${ }^{23}$ identify a dilemma that may limit the development of new elastocaloric SMAs. Figure 2 shows that a correlation exists between the latent heat and the hysteresis width. It is difficult to obtain the desired combination

\section{Functional stability}

The lowest functional degradation of the material is very important for any application. For example, a pseudoelastic SMA would have to tolerate $>15$ million loading/unloading cycles in a cooling device operating at $0.2 \mathrm{~Hz}$ over five years with $50 \%$ average utilization. Research on quaternary NiTi-based SMAs revealed a pronounced effect of crystallographic compatibility on the hysteresis width and reversibility (i.e., functional stability in thermally induced phase transformations) ${ }^{26}$ The crystallographic compatibility conditions can be derived from the transformation matrixes between austenite and martensite and are thus purely geometric. For crystallographic compatibility ${ }^{31}$ (where the two phases fit without the need for slip or for establishing stressed transition layers), the middle eigenvalue of the transformation matrix $\lambda_{2}$ must equal one. This can be achieved by fine-tuning the composition. This $\lambda_{2}=1$ condition affects the heights of the energy barriers between the transforming phases, which determine the width of the transformation hysteresis.

A higher degree of compatibility-so-called supercompatibility — can be obtained by additionally fulfilling other conditions that promote good fit and thus provide an even higher degree of flexibility, as was first demonstrated for $\mathrm{Zn}_{45} \mathrm{Au}_{30} \mathrm{Cu}_{25}$. ${ }^{32}$ The influence of supercompatibility on functional and structural fatigue in stress-induced austenite-martensite

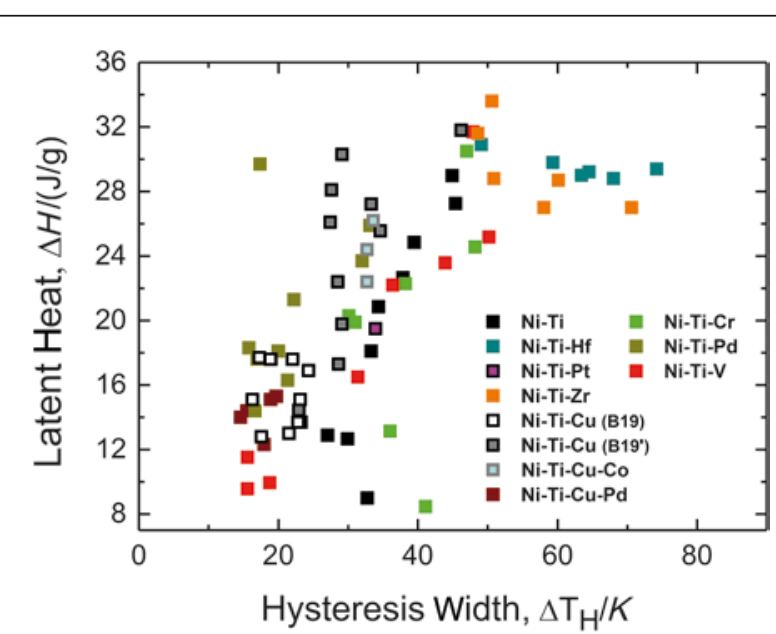

Figure 2. Key parameters for ferroic cooling-hysteresis width and latent heat. ${ }^{23}$ Alloy design requires good compromise (small hysteresis width and large latent heat) between both factors. 
transformations has been demonstrated for $\mathrm{TiNiCuCo}^{19}$ and $\mathrm{ZnAuCu},{ }^{33}$ and it was recently reviewed in Reference 31 . The composition $\mathrm{Ti}_{54.7} \mathrm{Ni}_{30.7} \mathrm{Cu}_{12.3} \mathrm{Co}_{2.3}$ showed no measurable fatigue for 10 million pseudoelastic $\operatorname{cycles}^{19}$ (Figure 3). This indicates that supercompatibility between austenite and martensite has an important influence on the fatigue behavior. From investigations of different $\mathrm{TiNiCu}$ and TiNiCuCo alloys with a $\lambda_{2}$-value close to one, the functional degradation behavior was found to also depend on microstructural parameters such as grain $\operatorname{size}^{34}$ and the nature of precipitates. ${ }^{19}$ It was found that $\mathrm{Ti}_{2} \mathrm{Cu}$ precipitates, which form in Ti-rich alloys, lead to a near-to-perfect reversibility and repeatability up to $>10$ million pseudoelastic cycles (Figure 3). The coherency of $\mathrm{Ti}_{2} \mathrm{Cu}$ precipitates in both phases, austenite and martensite, accounts for this excellent structural and functional property. These $\mathrm{Ti}_{2} \mathrm{Cu}$ precipitates guide the phase transformation in a well-defined manner and thus act like sentinels, assuring that the austenite-martensite transformation repeatedly follows the same path during each cycle. $^{19}$

\section{Ferroic cooling applications Bulk material-based cooling demonstrators}

The first successful large-scale elastocaloric cooling devices have been developed and demonstrated. ${ }^{35}$ The concept forming the basis of these developments is mechanical loading and unloading of SMA tube elements in compression and use of the absorbed and released latent heats to cool a hydraulic fluid. In contrast, the work presented in this article focuses on NiTibased ribbons or wires that are loaded in tension to develop scalable air-cooling devices. Inherently coupled to this design goal is the optimization of the process parameters in terms of cooling power and COP. This requires optimization of the amount of straining, the strain rate, and efficient synchronization between mechanical loading/unloading and heat transfer.

Extending the usual framework of calculating COP values, a graphical method similar to that used for conventional vapor compression cycles has been developed to illustrate relevant work and heat quantities in stress/ strain and temperature/entropy diagrams. ${ }^{36}$ This helps to predict optimized hybrid cycles by appropriately combining adiabatic and isothermal process branches. A comprehensive test rig was developed ${ }^{17,37-39}$ to experimentally validate process efficiency and to study the effects of material training/functional degradation on mechanical, caloric, and thermal properties (Figure 4). ${ }^{30}$ In the device shown in Figure 4a, a pseudoelastic NiTi ribbon is subjected to mechanical cycling. During this process, it moves between two copper blocks, Figure 4b, which act as a heat sink (in contact with the SMA during straining) and a heat source (in contact during unloading

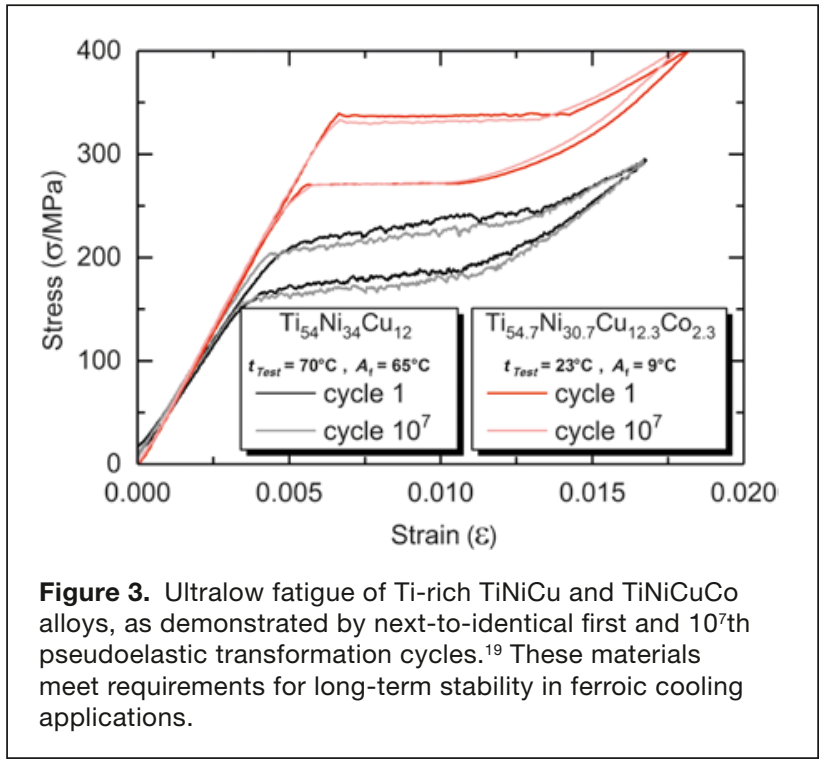

of the ribbon), respectively. Figure 4c exemplarily shows a color-coded IR image, which documents temperature changes in the ribbon and the two $\mathrm{Cu}$ blocks. A challenge for the design of future elastocaloric cooling devices is the implementation of these concepts into continuously operating units that outperform current vapor compression systems. The first ideas are currently being implemented into a demonstrator with a patent-pending drive element to map experimentally optimized processes. ${ }^{38}$ Simultaneously, due to the large number of parameters involved in the design, simulation tools have to be developed to narrow the design space.

\section{Thin-film cooling devices}

The implementation of elastocaloric cooling on a miniature scale involves opportunities and challenges. The use of freestanding elastocaloric films allows for efficient heat transfer between film and heat sink/-source, which is of critical 


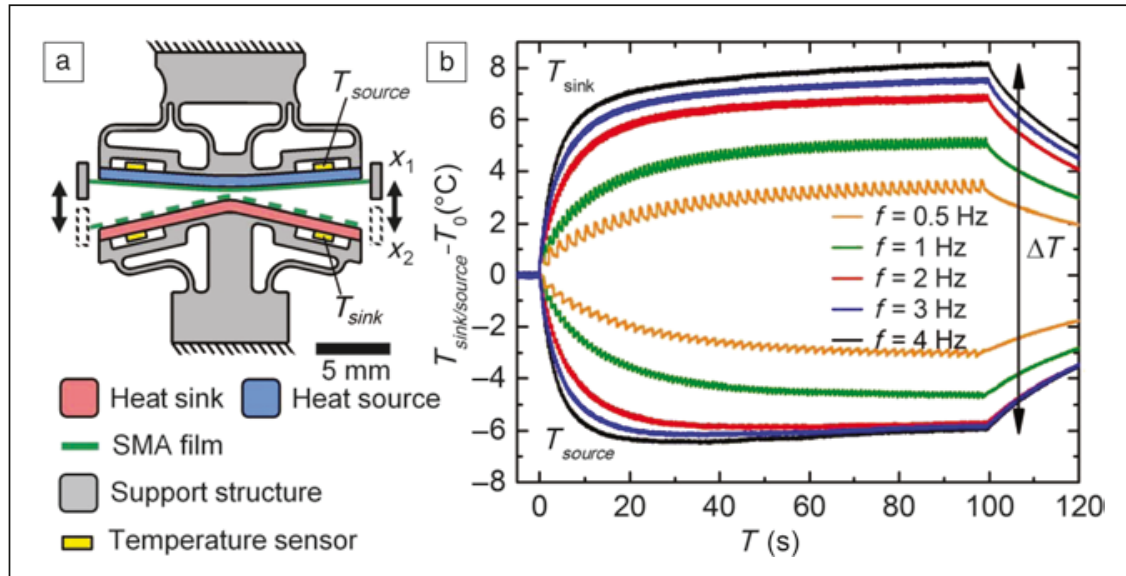

Figure 5. (a) Schematic of a miniature-scale cooling device. ${ }^{18}$ Moving the shape-memory alloy film periodically between the lower $\left(x_{2}\right)$ and upper $\left(x_{1}\right)$ position loads/unloads the film, and, thus, heat is generated/absorbed. Due to alternate switching of surface contacts in both positions, the moving film causes a directed heat flux from heat source to heat sink. (b) Experimentally observed temperature changes of the device for various operation frequencies reaching a maximum temperature span of $14 \mathrm{~K}$.

\section{Summary, outlook, and challenges}

Elastocaloric cooling principles have high potential for applications because (1) SMAs can provide high cooling efficiency, (2) they represent alternative engineering solutions for conventional cooling processes, and (3) both miniature and large-scale cooling units can be realized. Progress was recently achieved in alloy design where new NiTi-based SMAs with high cooling efficiencies and excellent fatigue lives were developed. These materials have to be studied to determine how they perform in actual cooling devices, and how process conditions can be optimized. For the development of new cooling devices, it may be necessary to extend the temperature range where the elastocaloric effect can be accessed. This will involve the development of cascaded cooling units where SMAs with different operating temperature ranges are combined.

importance for high cooling performance. However, parasitic heat fluxes are difficult to control in small systems, and system integration is often challenging. In addition, localized phase-transformation events may affect heat transport. ${ }^{13,40,41}$ One important feature of film-based SMAs is that they allow for almost pure tensile loading even when subjected to outof-plane deflection.

Figure 5a shows an example of a miniature cooling device where this loading principle is applied. Heating and cooling power is separated by bringing the SMA film alternatingly in contact with the heat source during unloading, upper position $x_{1}$, and with the heatsink during loading, lower position $x_{2}$. Switching heat fluxes and loading the film in one single mechanism makes the setup simple, which is crucial for miniaturization. A typical elastocaloric cycle includes moving the film up and down, which results in stepwise transfer of heat from the heat source to the heat sink. Thus, the temperature $T_{\text {source }}$ decreases and $T_{\text {sink }}$ increases, as can be seen in Figure $5 \mathrm{~b}$ for various operation frequencies.

The device reaches a maximum temperature span $\Delta T$ of $14 \mathrm{~K}$ at room temperature, a maximum cooling power of $18 \mathrm{Wg}^{-1}$, and a maximum COP of 6 at zero thermal load neglecting actuation efficiency. Keeping in mind that the film sample has a mass of only $0.015 \mathrm{~g}$, the results in Figure $5 \mathrm{~b}$ highlight the potential of small-scale elastocaloric cooling devices. Furthermore, it has been shown that work recovery can minimize the required mechanical power input in film-based cooling devices. ${ }^{18,42}$ For practical applications, the heat-source element can be interconnected to a thermal load (e.g., an electronic chip or a heat-transfer fluid). Currently, the elastocaloric cooling device is limited by the adiabatic temperature span of the SMA material, ${ }^{43}$ in this case, a NiTiCuCo alloy. This limit can be overcome by cascading several SMA films with tailored phase transition temperatures in a stack.

\section{Acknowledgments}

The authors acknowledge the support of the Priority Program 1599 Ferroic Cooling funded by the Deutsche Forschungsgemeinschaft DFG (Projects EG101/29-2, SE704/2-2, QU 146/22-2, and K02953/5-2).

\section{References}

1. S. Fähler, U.K. Rößler, O. Kastner, J. Eckert, G. Eggeler, H. Emmerich, P. Entel, S. Müller, E. Quandt, K. Albe, Adv. Eng. Mater. 14, 10 (2012).

2. L. Mañosa, A. Planes, M. Acet, J. Mater. Chem. A 1, 4925 (2013).

3. E. Bonnot, R. Romero, L. Mañosa, E. Vives, A. Planes, Phys. Rev. Lett. 100, 125901 (2008).

4. S. Miyazaki, K. Otsuka, ISIJ Int. 29, 353 (1989).

5. K. Otsuka, X. Ren, Prog. Mater. Sci. 50, 511 (2005).

6. E. Hornbogen, in Advanced Structural and Functional Materials, W.G.J. Bunk, Ed. (Springer Verlag, Köln, Germany, 1991), p. 133.

7. W. Goetzler, R. Zogg, J. Young, C. Johnson, "Energy Savings Potential and RD\&D Opportunities for Non-Vapor Compression HVAC Technologies" (Prepared for the US Department of Energy by Navigant Consulting Inc., 2014).

8. K. Bhattacharya, Microstructure of Martensite: Why It Forms and How It Gives Rise to the Shape-Memory Effect (Oxford University Press, Oxford, UK, 2004).

9. S. Qian, Y. Geng, Y. Wang, J. Ling, Y. Hwang, R. Radermacher, I. Takeuchi, J. Cui, Int. J. Refrig. 64, 1 (2016).

10. A. Wieczorek, J. Frenzel, M. Schmidt, B. Maaß, S. Seelecke, A. Schütze, G. Eggeler, Funct. Mater. Lett. 10, 1740001 (2017).

11. H. Sehitoglu, Y. Wu, E. Ertekin, Scr. Mater. (2017), doi.org/10.1016/j.scriptamat. 2017.05.017.

12. Y. Wu, E. Ertekin, H. Sehitoglu, Acta Mater. 135, 158 (2017).

13. H. Ossmer, F. Lambrecht, M. Gultig, C. Chluba, E. Quandt, M. Kohl, Acta Mater. 81, 9 (2014).

14. Y. Kim, M.-G. Jo, J.-W. Park, H.-K. Park, H.N. Han, Scr. Mater. 144, 48 (2018).

15. D. Luo, Y. Feng, P. Verma, Energy 130, 500 (2017).

16. J. Tusek, K. Engelbrecht, D. Eriksen, S. Dall'Olio, J. Tusek, N. Pryds, Nat. Energy 1, 16134 (2016)

17. M. Schmidt, A. Schütze, S. Seelecke, Int. J. Refrig. 54, 88 (2015).

18. H. Ossmer, F. Wendler, M. Gueltig, F. Lambrecht, S. Miyazaki, M. Kohl, Smart. Mater. Struct. 25, 085037 (2016).

19. C. Chluba, W.W. Ge, R.L. de Miranda, J. Strobel, L. Kienle, E. Quandt, M. Wuttig, Science 348, 1004 (2015)

20. M. Rahim, J. Frenzel, M. Frotscher, J. Pfetzing-Micklich, R. Steegmüller, M. Wohlschlögel, H. Mughrabi, G. Eggeler, Acta Mater. 61, 3667 (2013).

21. T. Waitz, V. Kazykhanov, H.P. Karnthaler, Acta Mater. 52, 137 (2004)

22. R. Delville, B. Malard, J. Pilch, P. Sittner, D. Schryvers, Int. J. Plast. 27, 282 (2011). 
23. J. Frenzel, A. Wieczorek, I. Opahle, B. Maaß, R. Drautz, G. Eggeler, Acta Mater. 90, 213 (2015).

24. J. Frenzel, E.P. George, A. Dlouhy, C. Somsen, M.F.X. Wagner, G. Eggeler, Acta Mater. 58, 3444 (2010).

25. J. Cui, Y.S. Chu, O.O. Famodu, Y. Furuya, J. Hattrick-Simpers, R.D. James, A. Ludwig, S. Thienhaus, M. Wuttig, Z.Y. Zhang, I. Takeuchi, Nat. Mater. 5, 286 (2006)

26. R. Zarnetta, R. Takahashi, M.L. Young, A. Savan, Y. Furuya, S. Thienhaus, B. Maass, M. Rahim, J. Frenzel, H. Brunken, Y.S. Chu, V. Srivastava, R.D. James, I. Takeuchi, G. Eggeler, A. Ludwig, Adv. Funct. Mater. 20, 1917 (2010).

27. S. Jaeger, B. Maaß, J. Frenzel, M. Schmidt, S. Seelecke, O. Kastner, G. Eggeler, Int. J. Mater. Res. 106, 1029 (2015)

28. Z.Y. Zhang, R.D. James, S. Müller, Acta Mater. 57, 4332 (2009).

29. J.M. Ball, R.D. James, Philos. Trans. R. Soc. A 338, 389 (1992)

30. M. Schmidt, J. Ullrich, A. Wieczorek, J. Frenzel, A. Schütze, G. Eggeler, S. Seelecke, Shape Mem. Superelast. 1, 132 (2015).

31. H. Gu, L. Bumke, C. Chluba, E. Quandt, A.D. James, Mater. Today, https://doi. org/10.1016/i.mattod.2017.10.002.

32. Y.T. Song, X. Chen, V. Dabade, T.W. Shield, R.D. James, Nature 502, 85 (2013).

33. X.Y. Ni, J.R. Greer, K. Bhattacharya, R.D. James, X. Chen, Nano Lett. 16 7621 (2016)

34. C. Chluba, W. Ge, T. Dankwort, C. Bechtold, R.L. de Miranda, L. Kienle, M. Wuttig, E. Quandt, Philos. Trans. R. Soc. A 374 (2016).

35. S.X. Qian, Y.L. Geng, Y. Wang, J. Muehlbauer, J.Z. Ling, Y.H. Hwang, R. Radermacher, I. Takeuchi, Sci. Technol. Built Environ. 22, 500 (2016).

36. M. Schmidt, A. Schütze, S. Seelecke, 2013 Proc. ASME Conf. Smart Mater. Adaptive Struct. Intell. Syst., N. Johnson, Ed. (ASME, New York, 2014), p. V001T04A014. 37. M. Schmidt, A. Schütze, S. Seelecke, 2014 Proc. ASME Conf. Smart Mater. Adaptive Struct. Intell. Syst., A. Zagrai, Ed. (ASME, New York, 2014), p. V002T04A013. 38. M. Schmidt, S.M. Kirsch, S. Seelecke, A. Schütze, Sci. Technol. Built Environ. 22, 475 (2016)

39. M. Schmidt, A. Schütze, S. Seelecke, APL Mater. 4, 064107 (2016).

40. H. Ossmer, C. Chluba, M. Gueltig, E. Quandt, M. Kohl, Shape Mem. Superelast. 1, 142 (2015)

41. F. Wendler, H. Ossmer, C. Chluba, E. Quandt, M. Kohl, Acta Mater. 136, 105 (2017).

42. F. Bruederlin, H. Ossmer, F. Wendler, S. Miyazaki, M. Kohl, J. Phys. D Appl. Phys. 50, 424003 (2017).

43. H. Ossmer, M. Kohl, Nat. Energy 1, 16159 (2016).

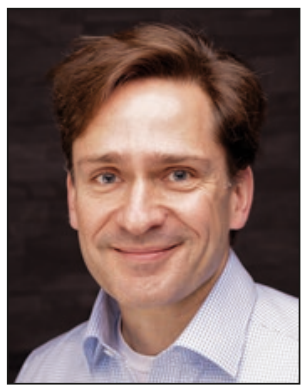

Jan Frenzel has been a senior researcher at Ruhr University Bochum, Germany, since 2007. He received his Dr.-Ing. degree in mechanical engineering from Ruhr University Bochum in 2005. He was a postdoctoral researcher at Ruhr University Bochum, Oak Ridge National Laboratory, and Northwestern University. In 2011, he received the Georg Sachs Award for his work on shape-memory alloys. He was a member of the board of directors of the International Organization on Shape Memory and Superelastic Technologies. His current research focuses on materials processing, shape-memory alloys, and superalloys. Frenzel can be reached by email at jan.a.frenzel@rub.de.

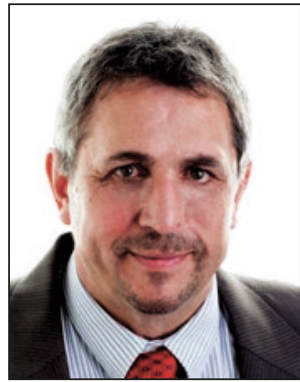

Gunther Eggeler has been professor of materials science at Ruhr University Bochum, Germany, since 1995. He studied materials science at Friedrich-Alexander-University Erlangen Nürnberg, Germany, where he obtained his Dipl-Ing degree in 1980 and Dr-Ing degree in 1984. He spent 10 years in Switzerland (1985 to 1987, 1991 to 1994, EPF Lausanne, Habilitation degree in 1993) and in the UK (1988-1990 as senior principal metallurgist in a private company). His current research focuses on elementary microstructural processes, which govern phase transformations and high-temperature plasticity. Eggeler can be reached by email at gunther.eggeler@rub.de.

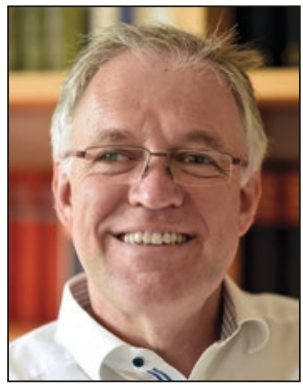

Eckhard Quandt is a professor in materials science at Kiel University, Germany. He studied physics at the University of Kiel and at the Technical University Berlin. He worked on smart materials from 1991 to 1999 in Karlsruhe, 1999 to 2006 at Caesar in Bonn, and since 2006 at the University of Kiel. From 2014 to 2016, he was dean of the Faculty of Engineering. He is a member of the "Materials Science" review board of the Deutsche Forschungsgemeinschaft and a member of the German National Academy of Science and Engineering. Quandt can be reached by email at eq@tf.uni-kiel.de.

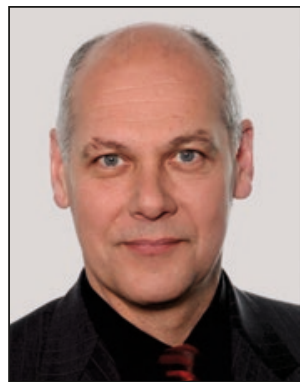

Stefan Seelecke is a full professor of systems engineering and materials science and engineering at Saarland University, Germany, where he directs the Intelligent Materials Systems Lab. He received his $\mathrm{PhD}$ degree in engineering science from Technical University Berlin, Germany, in 1995. After his Habilitation degree in 1999 he joined the Department of Mechanical and Aerospace Engineering at North Carolina State University in 2001. His research interests include the development of smart materials-based actuator and sensor systems, in particula (magnetic) shape-memory alloys, piezoelectrics, and electroactive polymers. Seelecke can be reached by email at stefan.seelecke@imsl.uni-saarland.de.

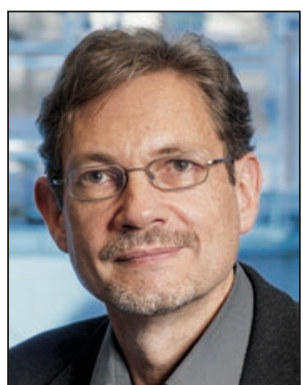

Manfred Kohl is a professor in the Faculty of Mechanical Engineering and head of the Department of Smart Materials and Devices at the Institute of Microstructure Technology at Karlsruhe Institute of Technology (KIT), Germany. He received his $\mathrm{PhD}$ degree in physics from the University of Stuttgart, Germany. He worked as an IBM postdoctoral fellow at the T.J. Watson Research Center in Yorktown Heights, and subsequently joined KIT. His current research focuses on multiferroic materials and corresponding microsystems, as well as multimaterial micro- and nanotechnologies. Kohl can be reached by email at manfred.kohl@kit.edu.

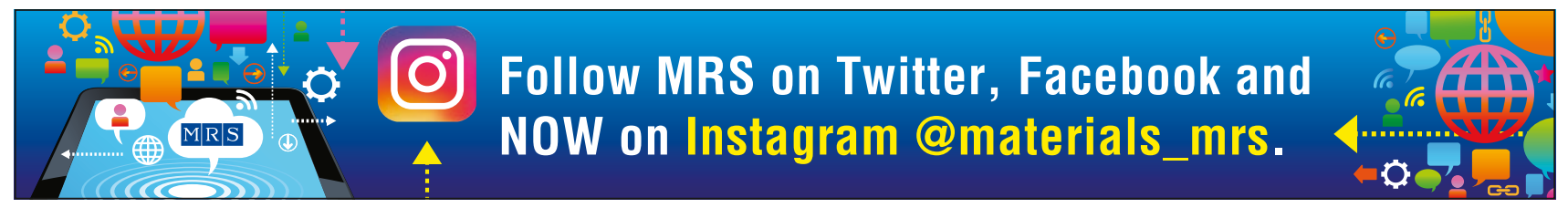

